\title{
8.5 RADiation BEAMing IN PULSARS
}

\author{
V. CANUTO \\ Institute for Space Studies,
}

Goddard Space Flight Center, NASA, New York, N.Y., U.S.A.

It is usually considered that the beaming of the radiation coming out of a pulsar has to be strictly connected with the mechanism producing the radiation itself. We want to show that even when the emitting mechanism gives rise to an isotropically distributed radiation, the presence of a strong magnetic field will automatically beam the radiation preferentially along the magnetic field line rather than in any other direction. We have computed the Compton scattering and from that the opacity $K_{H}$ ( $K_{0}$ is the opacity for zero field). In Figure 1 the ratio $K_{H} / K_{0}$ is given vs. $\theta$, the angle between the propagation vector and the magnetic field axis. $H_{q}$ is a critical magnetic field numerically equal to $4.41 \times 10^{13} \mathrm{G} ; N_{e}$ is the electron density. For the ordinary wave the opacity is reduced at $\theta=0$, while it is unaffected at $\theta=\pi / 2$ where $K_{H} \rightarrow K_{0}$.

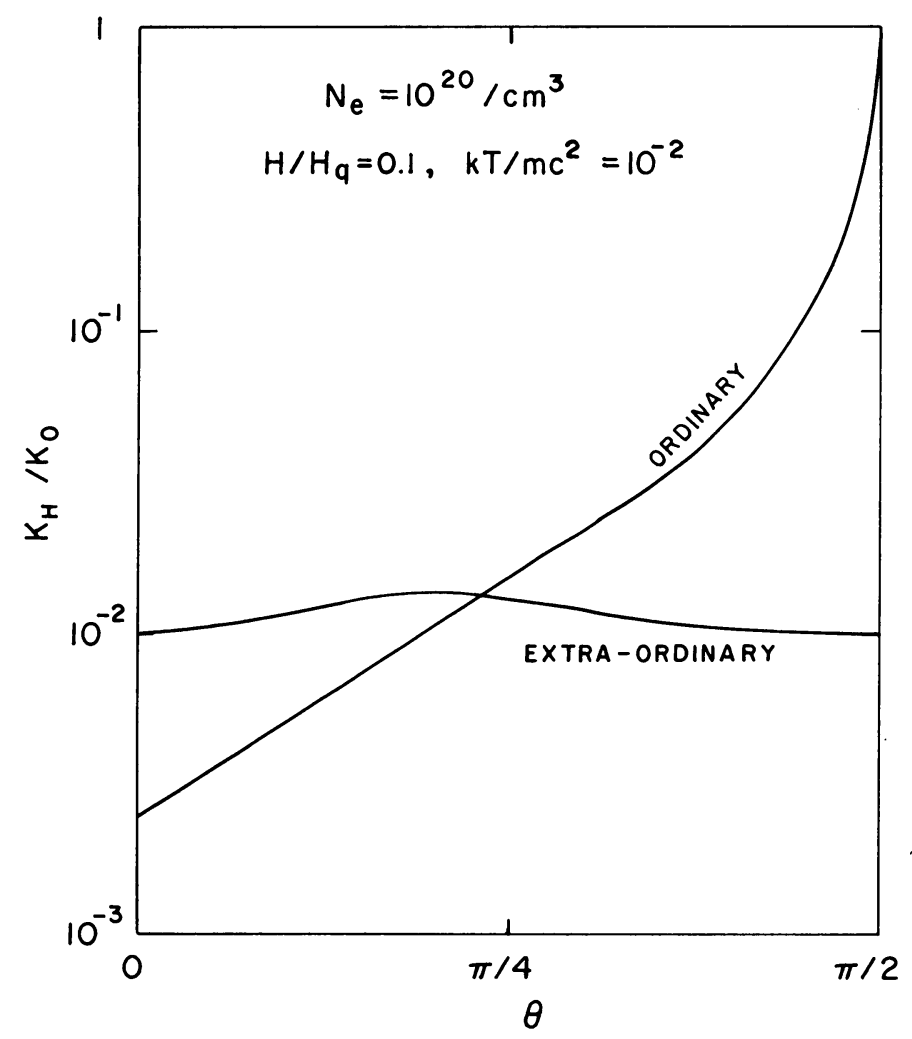

Fig. 1. 
Even at $\theta=\pi / 4$ the ratio $K_{H} / K_{0}$ is still $\simeq 10^{-2}$, and a good beaming is still present. The values of the parameters are proper for a neutron star surface. It is to be noticed that the ratio $K_{H} / K_{0}$ is of the order of $\left(\omega / \omega_{H}\right)^{2}$ or $\left[\left(k T / m c^{2}\right) /\left(H / H_{q}\right)\right]^{2}$. One therefore can conclude that the presence of a magnetic field itself assures the beaming of radiation along the field lines. 